\title{
Comparison of efficacy and safety between simultaneous integrated boost intensity-modulated radiotherapy and standard-dose intensity-modulated radiotherapy in locally advanced esophageal squamous cell carcinoma: a retrospective study
}

\author{
Wang Lan ${ }^{1} \cdot$ Liu Lihong $^{1} \cdot$ Han Chun $^{1} \cdot$ Liu Shutang $^{1} \cdot$ Wang Qi $^{1} \cdot$ Xu Liang $^{1} \cdot$ Li Xiaoning $^{1} \cdot$ Liu Likun $^{1}$
}

Received: 29 May 2021 / Accepted: 16 December 2021 / Published online: 14 January 2022

(c) The Author(s) 2022

\begin{abstract}
Objective This study aimed to evaluate the efficacy and safety of simultaneous integrated boost intensity-modulated radiotherapy (SIB-IMRT) versus standard-dose intensity-modulated radiotherapy (SD-IMRT) in the treatment of locally advanced esophageal squamous cell carcinoma.

Methods From July 2003 to March 2014, 1748 patients in a single center who received definitive chemoradiotherapy were included in the analysis. A total of 109 patients who underwent SIB-IMRT and fulfilled all inclusion and exclusion criteria were identified as the study group. A total of 266 patients who underwent SD-IMRT (60 Gy/30 fractions, 2 Gy/fraction, 1 time/day, 5 times/week) during the same period were selected as the control group. Propensity score matching (PSM) was used to balance the baseline characteristics. Survival status, treatment failure mode, and the occurrence of adverse events were compared between the two groups.

Results There were more women and more cervical and upper thoracic cancers $(P=0.038,<0.001$, respectively) in the SIB-IMRT group before case matching. The median progression-free survival (PFS) in the SD-IMRT and SIB-IMRT groups was 22 and 19 months, respectively, and the median overall survival duration was 24 and 22 months, respectively, with $\chi^{2}=0.244$ and $P=0.621$. After PSM of $1: 1,138$ patients entered the final analysis ( 69 cases from each group). The median PFS of the SD-IMRT group and the SIB-IMRT group was 13 and 18 months, respectively, with $\chi^{2}=8.776$ and $P=0.003$. The 1-, 3-, and 5-year overall survival rates were 66.7, 21.7, and 8.7\% and 65.2, 36.2, and 27.3\%, respectively, and the median overall survival duration was 16 and 22 months, respectively, with $\chi^{2}=5.362$ and $P=0.021$. Treatment failure mode: 5-year local regional recurrence rates of SD-IMRT and SIB-IMRT were 50.7 and 36.2\%, respectively, with $\chi^{2}=2.949$ and $P=0.086$. The 5-year distant metastasis rates of the two groups were 36.2 and $24.6 \%$, respectively, with $\chi^{2}=2.190$ and $P=0.139$. Adverse events: 3 patients experienced grade $4-5$ toxicity $(2.2 \%)$, including one case of grade 4 radiation esophagitis and two cases of grade 5 radiation pneumonitis, all in the SD-IMRT group; 14 patients experienced grade 3 adverse events $(10.1 \%)$, primarily including radiation esophagitis, radiation pneumonitis, and hematological toxicity. Conclusion The technique of SIB-IMRT was safe and reliable compared with SD-IMRT. In addition, SIB-IMRT had locoregional control advantages and potential survival benefits.
\end{abstract}

Keywords Esophageal neoplasms - Radiotherapy · Fractionation · Simultaneous integrated boost intensity-modulated radiotherapy $\cdot$ Survival

Data availability This is an open access article under the terms of the Creative Commons Attribution License, which permits use, distribution, and reproduction in any medium, provided the original work is properly cited.

The authors are accountable for all aspects of this work and ensure that questions related to the accuracy or integrity of any part of the work were appropriately investigated and resolved.
Prof. Han Chun hanchun2006@126.com

1 Department of Radiation Oncology, The Fourth Hospital of Hebei Medical University, 050011 Shijiazhuang, China 


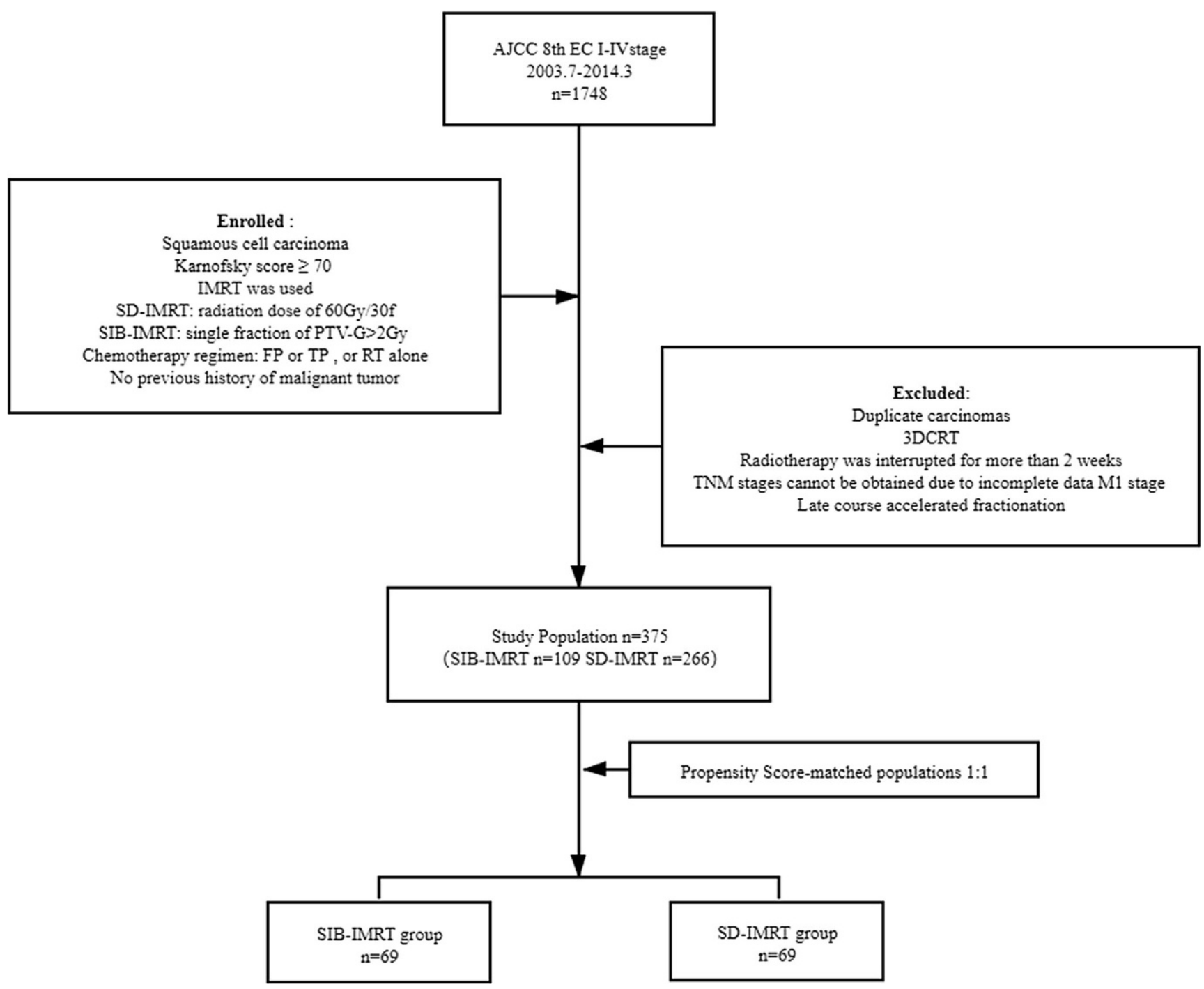

Fig. 1 Study flow diagram. FP fluorouracil and cisplatin, $T P$ paclitaxel and cisplatin

Simultaneous integrated boost intensity-modulated radiotherapy (SIB-IMRT) is an advantageous radiotherapy technique that can offer the unique capability of dose escalation by means of a simultaneous integrated boost (SIB). It can offer the advantage of delivering a higher dose to the primary tumor while conventional lower doses are used simultaneously to treat subclinical lesions or elective node regions. In simultaneous boosting, the total number of radiation therapy (RT) fractions is kept constant. SIB-IMRT has been successfully implemented to treat cancers of various regions such as the head and neck [1-4], prostate [5], and rectum [6, 7]. In the field of esophageal cancer, SIBIMRT technology also has application potential. However, because the esophagus is a lumen organ and adjacent to blood vessels, based on the risk of perforation and bleeding during the application of SIB-IMRT, the dose escalation range from the clinical target volume (CTV) to the gross tumor volume (GTV) is relatively small. Based on the re- search results of several phase I/II clinical trials [8-11], it is safe and feasible to use SIB-IMRT technology to escalate the total dose to a primary tumor to 59.92-70 Gy, with a single fraction dose of 2.14-2.8Gy. However, whether this technology could provide final local control or a survival benefit in the treatment of esophageal cancer still lacks sufficient research data [12-14]. Based on the aforementioned background, this study retrospectively analyzes a large number of esophageal cancer cases treated in a single center of our hospital. Patients who received SIB-IMRT were selected as the study group, and the control group was set using 1:1 propensity score matching (PSM). The purpose was to explore whether dose escalation using SIBIMRT technology might be beneficial in certain esophageal cancer patients. 
Table 1 Patient characteristics before and after PSM

\begin{tabular}{|c|c|c|c|c|c|c|}
\hline \multirow[t]{2}{*}{ Characteristics } & \multicolumn{3}{|c|}{ Before PSM $(n=375)$} & \multicolumn{3}{|c|}{ After PSM $(n=138)$} \\
\hline & $\begin{array}{l}\text { SD-IMRT group } \\
(n=266)\end{array}$ & $\begin{array}{l}\text { SIB-IMRT group } \\
(n=109)\end{array}$ & $P$-value ${ }^{\text {a }}$ & $\begin{array}{l}\text { SD-IMRT group } \\
(n=69)\end{array}$ & $\begin{array}{l}\text { SIB-IMRT group } \\
(n=69)\end{array}$ & $P$-value ${ }^{\mathrm{a}}$ \\
\hline \multicolumn{7}{|l|}{$\overline{\operatorname{Sex}(n)}$} \\
\hline Male & $179(67.3 \%)$ & $61(56.0 \%)$ & $0.038 *$ & $43(62.3 \%)$ & $43(62.3 \%)$ & 1.000 \\
\hline Female & $87(32.7 \%)$ & $48(44.0 \%)$ & & $26(37.7 \%)$ & $26(37.7 \%)$ & \\
\hline \multicolumn{7}{|l|}{ Age (years) } \\
\hline$\leq 70$ & $177(66.5 \%)$ & $91(83.5 \%)$ & 0.095 & $49(71.0 \%)$ & $56(81.2 \%)$ & 0.741 \\
\hline$>70$ & $89(33.5 \%)$ & $18(16.5 \%)$ & & $20(29.0 \%)$ & $13(18.8 \%)$ & \\
\hline Median (range) & $65(41 \sim 86)$ & $64(39 \sim 80)$ & & $65(41 \sim 84)$ & $64(39 \sim 80)$ & \\
\hline \multicolumn{7}{|c|}{ Lesion length (by barium meal, $\mathrm{cm}$ ) } \\
\hline$\leq 5.5 \mathrm{~cm}$ & $135(50.8 \%)$ & $67(61.5 \%)$ & 0.459 & $35(50.7 \%)$ & $43(62.3 \%)$ & 0.158 \\
\hline$>5.5 \mathrm{~cm}$ & $131(49.2 \%)$ & $42(48.5 \%)$ & & $34(49.3 \%)$ & $26(37.7 \%)$ & \\
\hline Median (range) & $5.6(0 \sim 13.9)$ & $5.5(2.4 \sim 10.2)$ & & $5.3(0-10.2)$ & $5.4(2.4 \sim 10.2)$ & \\
\hline \multicolumn{7}{|l|}{ Tumor site (n) } \\
\hline Cervical & $8(3.0 \%)$ & $17(15.6 \%)$ & $<0.001 *$ & $6(8.7 \%)$ & $7(10.1 \%)$ & 0.469 \\
\hline Upper & $79(29.7 \%)$ & $42(38.5 \%)$ & & $20(29.0 \%)$ & $25(36.2 \%)$ & \\
\hline Middle & $143(53.8 \%)$ & $42(38.5 \%)$ & & $38(55.1 \%)$ & $29(42.0 \%)$ & \\
\hline Lower & $36(13.5 \%)$ & $8(7.4 \%)$ & & $5(7.2 \%)$ & $8(11.6 \%)$ & \\
\hline \multicolumn{7}{|l|}{$T$ stage $(n)$} \\
\hline $\mathrm{T}_{1+2}$ & $41(15.4 \%)$ & $25(22.9 \%)$ & 0.221 & $12(17.4 \%)$ & $14(20.3 \%)$ & 0.904 \\
\hline $\mathrm{T}_{3}$ & $57(21.4 \%)$ & $21(19.3 \%)$ & & $14(20.3 \%)$ & $14(20.3 \%)$ & \\
\hline $\mathrm{T}_{4}$ & $168(63.2 \%)$ & $63(57.8 \%)$ & & $43(62.3 \%)$ & $41(59.4 \%)$ & \\
\hline \multicolumn{7}{|l|}{$N$ stage $(n)$} \\
\hline $\mathrm{N}_{0}$ & $22(8.3 \%)$ & $15(13.8 \%)$ & 0.156 & $3(4.3 \%)$ & $9(13.0 \%)$ & 0.180 \\
\hline $\mathrm{N}^{+}$ & $244(91.7 \%)$ & $94(86.2 \%)$ & & $66(95.7 \%)$ & $60(87.0 \%)$ & \\
\hline \multicolumn{7}{|l|}{$G T V$ volume $\left(\mathrm{cm}^{3}\right)$} \\
\hline$\leq 45 \mathrm{~cm}^{3}$ & $125(47.0 \%)$ & $61(56.0 \%)$ & 0.054 & $34(49.3 \%)$ & $34(49.3 \%)$ & 0.875 \\
\hline$>45 \mathrm{~cm}^{3}$ & $141(53.0 \%)$ & $48(44.0 \%)$ & & $35(50.7 \%)$ & $35(50.7 \%)$ & \\
\hline Median (range) & $47.9(1.8 \sim 189.2)$ & $41.2(3.8 \sim 174.7)$ & & $46.3(7.9 \sim 171.5)$ & $45.2(8.4 \sim 174.7)$ & \\
\hline \multicolumn{7}{|l|}{ Treatment regimen } \\
\hline RT alone & $118(44.4 \%)$ & $59(54.1 \%)$ & 0.119 & $35(50.7 \%)$ & $32(46.4 \%)$ & 0.902 \\
\hline $\begin{array}{l}\text { Sequential Chemo- } \\
\text { radiotherapy }\end{array}$ & $54(20.3 \%)$ & $23(21.1 \%)$ & & $14(20.3 \%)$ & $15(21.7 \%)$ & \\
\hline$C C R T$ & $94(35.3 \%)$ & $27(24.8 \%)$ & & $20(29.0 \%)$ & $22(31.9 \%)$ & \\
\hline \multicolumn{7}{|c|}{ Prescription dose $(G y)^{\mathrm{b}}$} \\
\hline Range & 60 & $\begin{array}{l}59.92 \sim 66 / \\
50.4 \sim 60.00^{\mathrm{b}}\end{array}$ & - & 60 & & $\begin{array}{l}59.92 \sim 66.00 / \\
50.4 \sim 60.00^{\mathrm{b}}\end{array}$ \\
\hline Median & 60 & $63 / 57$ & - & 60 & $64 / 59$ & - \\
\hline \multicolumn{7}{|l|}{ Fractions } \\
\hline Range & 30 & $27 \sim 31$ & - & 30 & $27 \sim 31$ & - \\
\hline Median & 30 & 30 & - & 30 & 30 & - \\
\hline
\end{tabular}

$P S M$ propensity score matching, $P T V-G$ the planning target volume of GTV-p and GTV-n, $P T V$ - $C$ the planning tartget volume of CTV ${ }^{\mathrm{a}} \chi^{2}$ or two-independent-sample tests

${ }^{b}$ Before PSM: the prescription dose range of PTV ${ }_{-G}$ was $59.92-66.00 \mathrm{~Gy}$, median: $63.00 \mathrm{~Gy}$; the prescription dose range of PTV ${ }_{-C}$ was 50.4-60.00 Gy, median: 57.00 Gy. After PSM: the prescription dose range of PTV-G was 59.92-66.00 Gy, median: 64Gy; the prescription dose range of PTV-C was 50.4-60.00 Gy, median: 59 Gy

*Statistically significant $p$-value 


\section{Materials and methods}

\section{Patients and eligibility criteria}

From July 2003 to March 2014, 1748 patients with esophageal cancer treated by definitive radiotherapy in our hospital were analyzed. The inclusion criteria consisted of the following: 1) squamous cell carcinoma confirmed by pathology; 2) a Karnofsky score $\geq 70$; 3) intensitymodulated radiotherapy technology was used; 4) for patients undergoing SIB-IMRT, the single fraction dose for the planning target volume (PTV) of the GTV region was $>2 \mathrm{~Gy}$, for those who received conventionally fractionated radiotherapy, the $60 \mathrm{~Gy} / 30$ fractions mode was selected as the standard-dose intensity-modulated radiotherapy (SDIMRT) for the control; 5) chemotherapy consisting of the FP (5-fluorouracil+cisplatin) or TP (paclitaxel+cisplatin) regimen was used or radiotherapy alone; and 6) no history of malignant tumor. The exclusion criteria consisted of the following: 1) multi-primary esophageal carcinoma; 2) conformal radiotherapy; 3) radiotherapy interruption for more than 2 weeks; 4) insufficient imaging data and unable to define the TNM stage; 5) M1 stage patients; and 6) latecourse accelerated hyperfractionated radiotherapy. After screening according to the inclusion and exclusion criteria, 375 cases met the enrollment conditions with 109 cases in the SIB-IMRT group and 266 cases in the SD-IMRT group. The study flow diagram is shown in Fig. 1.The clinical data and comparability tests of the two groups are shown in Table 1. The staging was based on the eighth edition of the American Joint Committee on Cancer (AJCC) clinical TNM (cTNM) staging standard for esophageal cancer.

\section{Radiation therapy}

All patients underwent computed tomography (CT)-based treatment simulation in the supine position, and 3-mm thick images were obtained throughout the entire neck, thorax, and upper abdomen. The scanned images were transferred to a three-dimensional (3D) planning system. The GTV, CTV, PTV, and normal organs at risk (OAR) were delineated layer by layer. The GTV included primary tumors $\left(G V_{-P}\right)$ and lymph node metastasis $\left(G V_{-n}\right)$. The $G V_{-P}$ included all esophageal tumors that were found using a CT scan, esophageal barium, endoscopy, endoscopic ultrasonography (EUS), and PET-CT. The $\mathrm{GTV}_{-n}$ was defined as any lymph node diagnosed as or highly suspected of being metastatic. The CTV of a primary tumor $\left(\mathrm{CTV}_{-\mathrm{P}}\right)$ was defined as the GTV -p $_{\text {p }}$ plus a 2-cm margin superiorly and inferiorly and a $0.5-\mathrm{cm}$ margin laterally along the esophagus. For the CTV of the lymph node $\left(\mathrm{CTV}_{-\mathrm{n}}\right)$, involved-field radiotherapy (IFI) was used for the majority of patients. However, if the primary tumor was in the cervical or upper thoracic esophagus, the $\mathrm{CTV}_{-n}$ encompassed the elective nodal area including the bilateral supraclavicular and upper mediastinal lymph node regions. The PTV of the clinical target volume $\left(\mathrm{PTV}_{-\mathrm{C}}\right)$ was generated by adding a $1-\mathrm{cm}$ margin craniocaudally, a $0.5-\mathrm{cm}$ margin laterally along the $\mathrm{CTV}_{-\mathrm{P}}$, and a uniform $0.5-\mathrm{cm}$ margin around $\mathrm{CTV}_{-\mathrm{n}}$. The PTV $_{-\mathrm{G}}$ was defined using the GTV $\left(\mathrm{GTV}_{-\mathrm{P}}+\mathrm{GTV}_{-\mathrm{n}}\right)$ plus a $0.3-0.5 \mathrm{~cm}$ margin. For patients undergoing SIBIMRT, the $\mathrm{PTV}_{-\mathrm{G}}$ and $\mathrm{PTV}_{-\mathrm{C}}$ received two prescription doses simultaneously. The lower dose was delivered to the $\mathrm{PTV}_{-\mathrm{C}}$, and the higher dose was escalated to the PTV -G. $_{\text {.G }}$ For patients undergoing SD-IMRT, only one prescription dose was delivered to the $\mathrm{PTV}_{\text {-c. }}$ A prescription dose was

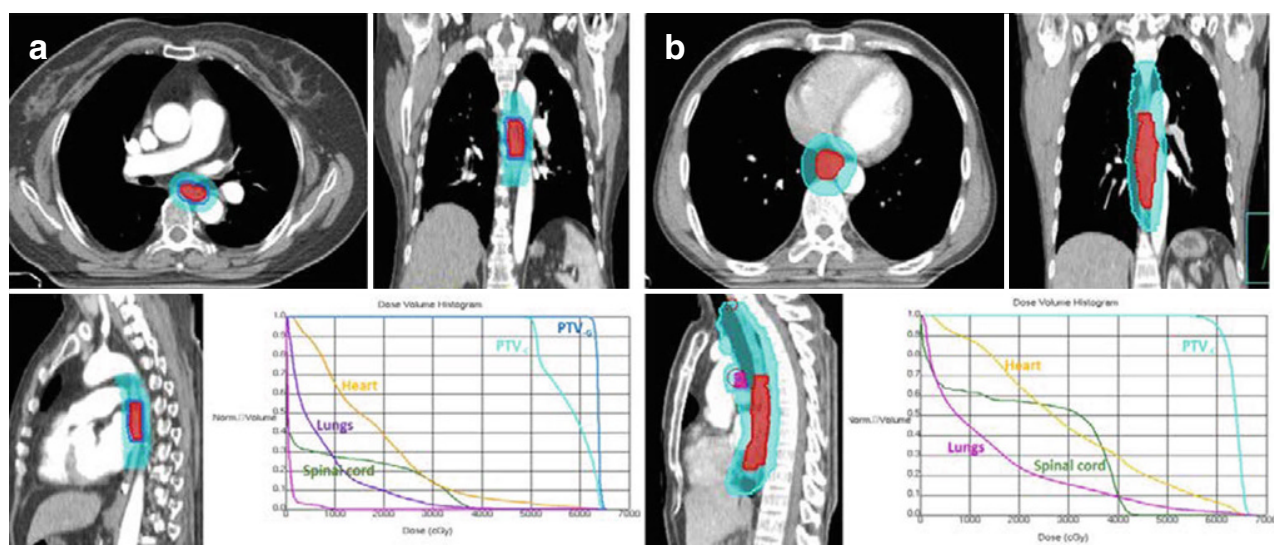

Fig. 2 The representative SIB-IMRT and SD-IMRT planning images with contours and the dose-volume histogram. a Target contour of SIBIMRT plan for a patient with ESCC, displayed on the axial, coronal, and sagittal planes through the primary tumor. Dose-volume histograms

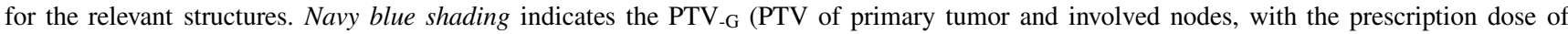

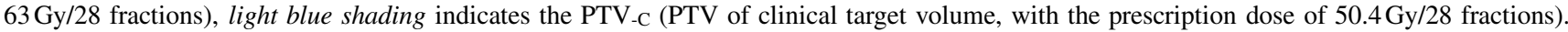
b Target contour of SD-IMRT plan for a patient with ESCC, displayed on the axial, coronal, and sagittal planes through the primary tumor.

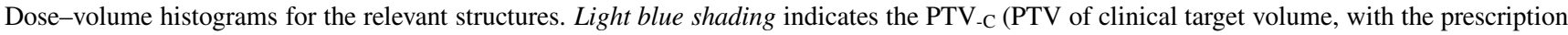
dose of $60 \mathrm{~Gy} / 30$ fractions) 
defined as $95 \%$ of the receiving dose of the PTV, with the difference of the internal target dose uniformity of $<5 \%$, and the internal target maximum dose point of $\leq 110 \%$. The OAR included the spinal cord, lungs, and the heart. The treatment plan generally required the entire lungs $\mathrm{V} 5 \leq 55-60 \%, \mathrm{~V} 20 \leq 25-30 \%$, and $\mathrm{V} 30 \leq 18 \%$; a mean heart dose of $\leq 26-30 \mathrm{~Gy}$; and a maximum spinal cord dose of $<45 \mathrm{~Gy}$. For the SIB-IMRT group, the prescribed doses were 50.4-60 Gy/27-31 fractions $(1.8-2.0 \mathrm{~Gy} /$ fraction) to the PTV $_{-\mathrm{C}}$ and 59.92-66 Gy/27-31 fractions (2.06-2.29 Gy/


The prescribed doses of the SD-IMRT group were $60 \mathrm{~Gy} / 30$ fractions, $2 \mathrm{~Gy} / \mathrm{fraction}, 1$ time/day, and 5 times/ week. The representative SIB-IMRT and SD-IMRT planning images with contours and the dose-volume histogram are shown in Fig. 2.

\section{Chemotherapy}

A total of 198 cases of the 375 patients received chemotherapy, including 54 cases of sequential chemoradiotherapy and 94 cases of concurrent chemoradiotherapy in the SDIMRT group, 23 cases of sequential chemoradiotherapy, and 27 cases of concurrent chemoradiotherapy in the SIBIMRT group. The chemotherapy regimen was FP or TP [15-18] with the following usage: cisplatin $75 \mathrm{mg} / \mathrm{m}^{2}$, divided into 3 to 5 days, 5 -FU $450-500 \mathrm{mg} / \mathrm{m}^{2} \times 5$ days (first to fifth days); or paclitaxel $135 \mathrm{mg} / \mathrm{m}^{2}$, the first day intravenously, with cisplatin $25 \mathrm{mg} / \mathrm{m}^{2} \times 3$ days (days 2,3 , and 4). Concurrent chemotherapy was given during the first and fourth or fifth weeks of radiotherapy.

\section{Observation endpoints}

The primary observational endpoint was long-term overall survival (OS), and the secondary endpoints were progression-free survival (PFS), treatment failure mode, and adverse events of grade $\geq 3$. Cox regression model was used for the multivariate analysis to evaluate the benefit value of SIB-IMRT.

\section{Statistical analysis and follow-up}

All statistical analyses were performed using the SPSS 22.0 software package (IMB Corp., Armonk, NY, USA). OS and PFS was assessed using the Kaplan-Meier method, and difference between the groups was assessed using the log-rank test. The patients who were lost to follow-up were calculated according to survival at the last follow-up. A Cox regression was used to analyze the prognostic factors. The case-control selection was according to the 1:1 principle, and cases were selected from the SD-IMRT group and matched with the SIB-IMRT group using the propensity score matching (PSM) module in the SPSS 22.0 software package (the biostatistical method of logistic regression was used), the matching variables included gender, age, tumor location, clinical T stage, N stage, TNM stage, GTV volume, and chemoradiotherapy combination mode of the two groups. The adopted caliper width was 0.02 and $P<0.05$ was considered statistically significant.

\section{Results}

\section{Patient characteristics}

There were 109 cases in the SIB-IMRT group and 266 cases in the SD-IMRT group. Before the PSM, the baseline characteristics of the two groups were different (Table 1). In the SIB-IMRT group, there were more women and more cervical and upper thoracic cancers $(P=0.038,<0.001$, respectively). A total of 69 pairs (138 cases) of patients were successfully matched after PSM, numbers of patients treated with SD-IMRT or SIB-IMRT year by year are shown in the Supplementary Table. The patient characteristics of the two

Table 2 Toxic events among 138 patients given SD-IMRT and SIB-IMRT

\begin{tabular}{|c|c|c|c|c|c|c|c|c|c|c|c|c|}
\hline \multirow[t]{2}{*}{$\overline{\mathrm{AEs}}$} & \multicolumn{5}{|c|}{ SD-IMRT } & \multicolumn{5}{|c|}{ SIB-IMRT } & \multirow[t]{2}{*}{$\overline{\chi^{2}}$} & \multirow{2}{*}{$\begin{array}{l}P \text { - } \\
\text { value }\end{array}$} \\
\hline & Grade 0 & Grade 1 & Grade 2 & Grade 3 & $\begin{array}{l}\text { Grade } 4 \\
\text { or } 5\end{array}$ & Grade 0 & Grade 1 & Grade 2 & Grade 3 & $\begin{array}{l}\text { Grade } 4 \\
\text { or } 5\end{array}$ & & \\
\hline $\begin{array}{l}\text { Radiation } \\
\text { esophagitis }\end{array}$ & 16 & 15 & 34 & 3 & 1 & 21 & 15 & 32 & 1 & 0 & 2.632 & 0.662 \\
\hline $\begin{array}{l}\text { Radiation } \\
\text { pneumonitis }\end{array}$ & 49 & 14 & 1 & 3 & 2 & 54 & 5 & 8 & 2 & 0 & 11.789 & 0.009 \\
\hline $\begin{array}{l}\text { Hematological } \\
\text { toxicity }\end{array}$ & 29 & 28 & 11 & 1 & 0 & 19 & 27 & 19 & 4 & 0 & 5.844 & 0.116 \\
\hline Nausea & 46 & 19 & 4 & 0 & 0 & 40 & 24 & 5 & 0 & 0 & 1.153 & 0.624 \\
\hline Vomit & 59 & 6 & 4 & 0 & 0 & 52 & 12 & 5 & 0 & 0 & 2.547 & 0.301 \\
\hline $\begin{array}{l}\text { Diarrhea/ } \\
\text { constipation }\end{array}$ & 64 & 4 & 1 & 0 & 0 & 66 & 3 & 0 & 0 & 0 & 1.161 & 0.718 \\
\hline
\end{tabular}


groups are shown in Table 1, and there was no significant difference between the two groups.

\section{Adverse events}

The main treatment-related adverse events of the two groups were acute radiation esophagitis, acute pneumonitis, and hematological toxicity. Details of the toxic effects are shown in Table 2. Among them, 3 patients experienced grade 4 to 5 toxic effects $(2.2 \%)$, including one case of grade 4 radiation esophagitis (stenosis, pain, severely affecting eating and life, and the patient received gastrointestinal nutrition tube implantation, intravenous anti-inflammatory, acid inhibition, mucosal protection, and nutritional support treatment), and two cases of grade 5 radiation pneumonitis (death 1 month after radiotherapy due to respiratory failure secondary to pulmonary infection), all in the SDIMRT group. Grade 3 adverse events occurred in 14 patients $(10.1 \%)$, including radiation esophagitis in four cases (SD-IMRT group, three cases [4.3\%]; SIB-IMRT group, one case $[1.4 \%]$ ); radiation pneumonitis in five cases (SDIMRT group, three cases [4.3\%]; SIB-IMRT group, two cases [2.9\%]); hematological toxicity in five cases (SDIMRT group, one case [1.4\%]; SIB-IMRT group, four cases $[5.8 \%])$. Due to this being a retrospective analysis,

a

Overall survival by radiotherapy modality before PSM

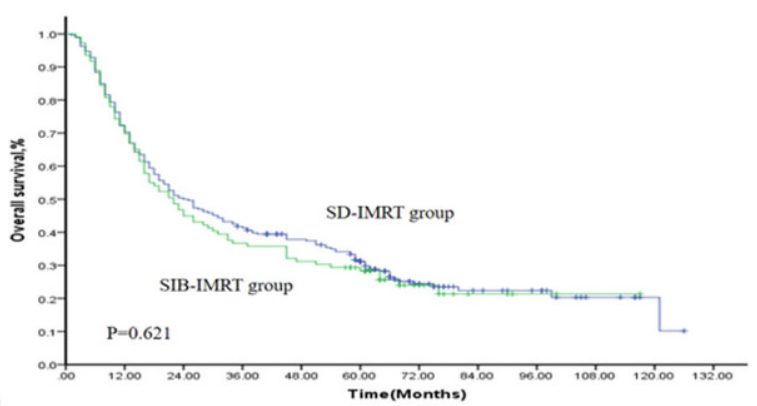

No. at risk

SD-IMIRT

SIB-IMRT



Progression-free survival by radiotherapy modality before PSM

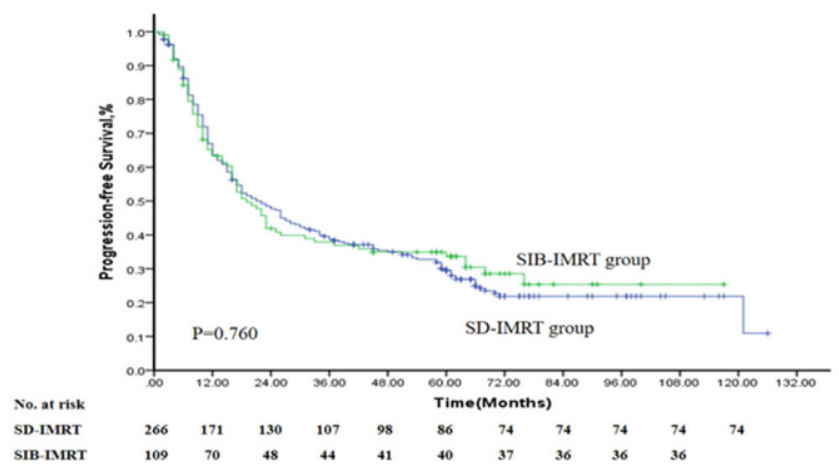

the study failed to obtain the occurrence of late adverse reactions in the two IMRT mode groups.

\section{Survival and treatment failure mode}

Until the date of follow-up, a total of 10 cases were lost to follow-up, with a follow-up rate of $97.3 \%$. Follow-up methods included telephone calls, letters, a hospital review, and visits. According to the Kaplan-Meier method, before case-control matching, the median PFS of the SD-IMRT and SIB-IMRT groups was 22 and 19 months, respectively, with $\chi^{2}=0.093$ and $P=0.760$. The 1-, 3-, and 5-year overall survival rates of the two groups were $70.3,41.3$, and $31.2 \%$ and $69.7,36.7$, and $28.3 \%$, respectively. The median overall survival time was 24 and 22 months, respectively, with $\chi^{2}=0.244$ and $P=0.621$. There was no significant difference in the PFS and OS between the two groups (Fig. 3a, c). After case matching, the median PFS of the two groups was 13 and 18 months, with $\chi^{2}=8.776$ and $P=0.003$. The $1-$, 3 -, and 5-year overall survival rates of the two groups were $66.7,21.7$, and $8.7 \%$ and $65.2,36.2$, and $27.3 \%$, respectively. The median OS time was 16 and 22 months with $\chi^{2}=5.362$ and $P=0.021$. The PFS and OS of the SIB-IMRT group were significantly better than those of the SD-IMRT group (Fig. 3b, d).

b

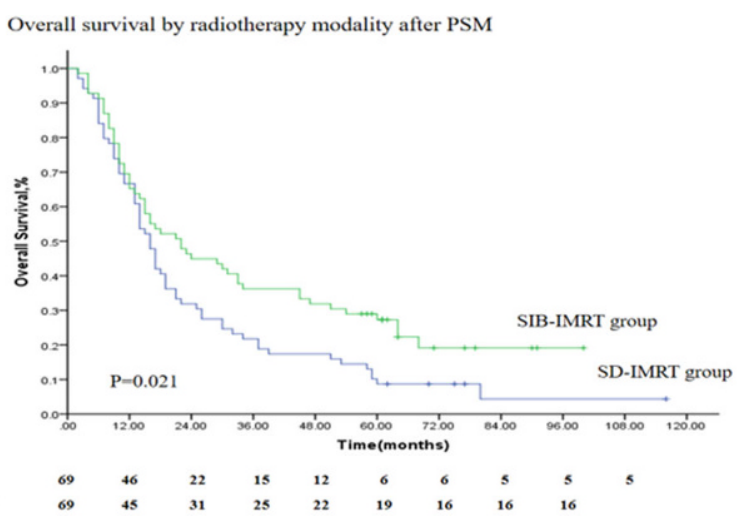

No. at risk SD-IMRT
SIB-IMRT

d

Progression-free survival by radiotherapy modality after PSM

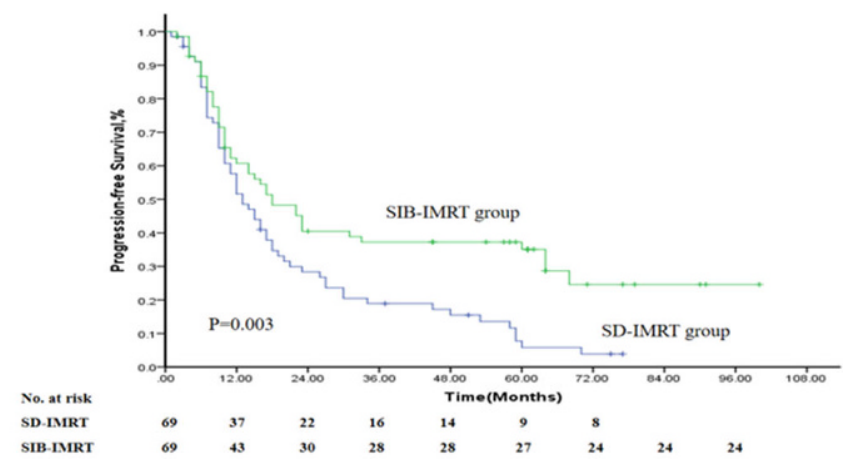

Fig. 3 Overall survival and progression-free survival of the study population. a Overall survival by radiotherapy modality before PSM; b overall survival by radiotherapy modality after PSM; c progression-free survival by radiotherapy modality before PSM; d progression-free survival by radiotherapy modality after PSM 
a Local Regional recurrence by radiotherapy modality after PSM



b Metastasis incidence by radiotherapy modality after PSM

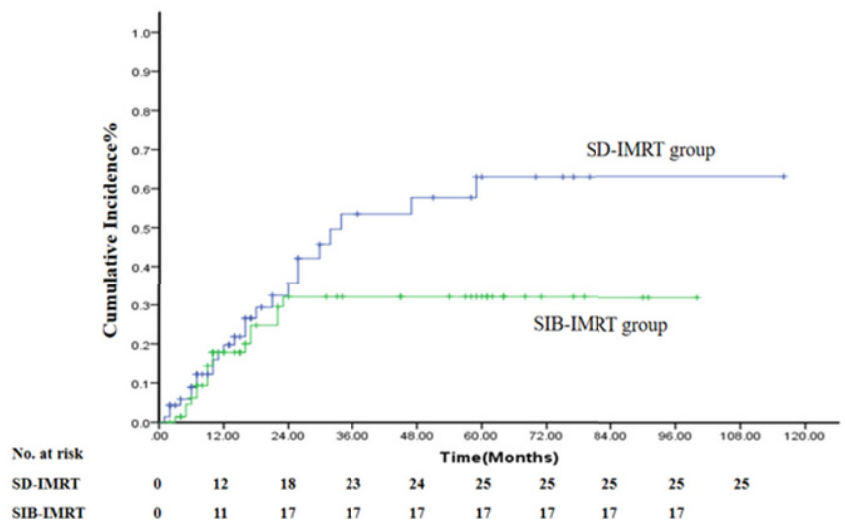

Fig. 4 Locoregional recurrence rate and metastasis incidence of the study population. a Locoregional recurrence by radiotherapy modality after PSM; b metastasis incidence by radiotherapy modality after PSM

Table 3 Univariate and multivariate Cox analyses of patients after PSM

\begin{tabular}{|c|c|c|c|c|c|c|c|c|}
\hline \multirow[t]{2}{*}{ Characteristic } & \multirow[t]{2}{*}{ Cases } & \multicolumn{4}{|c|}{ Univariate analysis } & \multicolumn{3}{|c|}{ Multivariate analysis } \\
\hline & & $\begin{array}{l}\text { MST } \\
\text { (months) }\end{array}$ & HR & $95 \% \mathrm{CI}$ & $P$-value & HR & $95 \% \mathrm{CI}$ & $P$-value \\
\hline \multicolumn{9}{|l|}{ Sex } \\
\hline Male & 86 & 16 & 1 & (Reference) & (Reference) & 1 & (Reference) & (Reference) \\
\hline Female & 52 & 22 & 0.573 & $0.387-0.847$ & $0.005 *$ & 0.546 & $0.368-0.811$ & $0.003^{*}$ \\
\hline \multicolumn{9}{|l|}{ Age (years) } \\
\hline$\leq 70$ & 105 & 18 & 1 & (Reference) & (Reference) & - & - & - \\
\hline$>70$ & 33 & 14 & 1.684 & $1.116-2.540$ & 0.013 & - & - & - \\
\hline \multicolumn{9}{|l|}{ Tumor site } \\
\hline Cervical/upper & 58 & 21 & 1 & (Reference) & (Reference) & - & - & - \\
\hline Middle/lower & 80 & 15 & 1.320 & $0.910-1.913$ & 0.143 & - & - & - \\
\hline \multicolumn{9}{|c|}{ Lesion length (barium meal, $\mathrm{cm}$ ) } \\
\hline$\leq 5.5 \mathrm{~cm}$ & 78 & 19 & 1 & (Reference) & (Reference) & - & - & - \\
\hline$>5.5 \mathrm{~cm}$ & 60 & 15 & 1.364 & $0.947-1.964$ & 0.096 & - & - & - \\
\hline \multicolumn{9}{|l|}{ T stage } \\
\hline $\mathrm{T}_{1+2}$ & 26 & 30 & 1 & (Reference) & (Reference) & 1 & (Reference) & (Reference) \\
\hline $\mathrm{T}_{3}$ & 28 & 15 & 2.420 & $1.319-4.441$ & $0.004 *$ & 2.830 & $0.528-5.241$ & $0.001^{*}$ \\
\hline $\mathrm{T}_{4}$ & 84 & 16 & 1.709 & $1.018-2.870$ & $0.043^{*}$ & 1.800 & $1.070-3.027$ & $0.027 *$ \\
\hline \multicolumn{9}{|l|}{ N stage } \\
\hline $\mathrm{N}_{0}$ & 12 & 45 & 1 & (Reference) & (Reference) & - & - & - \\
\hline$N_{+}$ & 126 & 16 & 2.242 & $1.042-4.823$ & 0.039 & - & - & - \\
\hline \multicolumn{9}{|l|}{ GTV volume } \\
\hline$\leq 45 \mathrm{~cm}^{3}$ & 68 & 22 & 1 & (Reference) & (Reference) & - & - & - \\
\hline$>45 \mathrm{~cm}^{3}$ & 70 & 14 & 1.462 & $1.015-2.106$ & 0.041 & - & - & - \\
\hline \multicolumn{9}{|l|}{ Radiotherapy modality } \\
\hline SD-IMRT & 69 & 16 & 1 & (Reference) & (Reference) & 1 & (Reference) & (Reference) \\
\hline SIB-IMRT & 69 & 22 & 0.655 & $0.454-0.946$ & $0.024 *$ & 0.606 & $0.417-0.878$ & $0.008^{*}$ \\
\hline \multicolumn{9}{|l|}{ Treatment regimen } \\
\hline RT alone & 67 & 17 & 1 & (Reference) & (Reference) & - & - & - \\
\hline $\begin{array}{l}\text { Sequential chemoradio- } \\
\text { therapy }\end{array}$ & 29 & 18 & 0.731 & $0.444-1.204$ & 0.218 & - & - & - \\
\hline CCRT & 42 & 16 & 1.071 & $0.709-1.617$ & 0.745 & - & - & - \\
\hline
\end{tabular}

*Statistically significant $p$-value 
A treatment failure mode analysis was performed for the matched cohort, and the cumulative locoregional recurrence (primary tumor recurrence and regional lymph node metastasis) and distant metastasis of the two groups are shown in Fig. 4. The 5-year local regional recurrence rates of SDIMRT and SIB-IMRT were 50.7 and $36.2 \%$, respectively, with $\chi^{2}=2.949$ and $P=0.086$. The 5-year distant metastasis rates of the two groups were 36.2 and $24.6 \%$, respectively, with $\chi^{2}=2.190$ and $P=0.139$.

\section{Analysis of the prognostic factors}

The Cox regression model was used to screen the prognostic factors of the matched data set. The covariates that entered the analysis included gender, age, tumor location, tumor length, T stage, N stage, GTV volume, radiotherapy mode (SD-IMRT vs. SIB-IMRT), and the combination mode of chemoradiotherapy (radiotherapy alone vs. sequential chemoradiotherapy vs. concurrent chemoradiotherapy). The final independent prognostic factors selected included gender, T staging, and the radiotherapy mode. SIBIMRT was a survival benefit factor compared with SDIMRT $(\mathrm{HR}=0.606, P=0.008$; Table 3$)$.

\section{Discussion}

Radiation at a dose of $50 \mathrm{~Gy}$ combined with concurrent chemotherapy is the standard therapy for patients with localized carcinoma of the esophagus who are selected for nonsurgical treatment based on the intergroup trial RTOG 8501 [19, 20]. However, the dose of 50Gy is relatively low compared with radiation doses used in curative CRT schemes for other carcinomas such as lung cancer and head and neck cancer, and higher locoregional control rates are achieved in these tumors $[21,22]$. In an attempt to improve locoregional control, the randomized RTOG INT 0123 trial [23] compared CRT using a high dose $(64.8 \mathrm{~Gy} / 1.8 \mathrm{~Gy})$ with a standard dose (SD, 50.4Gy/1.8 Gy) combined with concurrent chemotherapy. There was no significant difference in locoregional failure (52 vs. $56 \%$ ) or in the 2-year overall survival (OS; $31 \%$ vs. $40 \%$ ) between the high- and standard-dose arms. Since then, radiation of 50.0-50.4 Gy has come to be considered as the standard dose of definitive chemoradiation (dCRT) for esophageal cancer. After entering the era of precision radiotherapy, a recent randomized controlled study (ARTDECO) [14] also showed that in dCRT for esophageal cancer, a radiation dose escalation up to $61.6 \mathrm{~Gy}$ to the primary tumor did not result in a significant increase in local control over $50.4 \mathrm{~Gy}$, and the absence of a dose effect was observed in both adenocarcinoma (AC) and squamous cell carcinoma (SCC). Hence, most centers are actually de-escalating the radiation dose worldwide.
However, for esophageal cancer, local control is always the key to success of treatment, especially for patients undergoing nonsurgical treatment. In the RTOG 8501 trial, the locoregional failure rate after dCRT was high (47\%), and this was also demonstrated in several other large dCRT series [23-26]. Based on the results of a meta-analysis [27], a prescription dose $\geq 60 \mathrm{~Gy}$ was found to be more conducive to improving the overall survival and local control of esophageal squamous cell carcinoma in Asian countries. In a retrospective analysis of our center [28], high-dose concurrent chemoradiotherapy (cCRT) of $60 \mathrm{~Gy}$ produced long-term OS and LC benefits compared with the standarddose cCRT of 50.4-54.0 Gy, with 10-year OS rates of 24 and $13.3 \%$, respectively, and 10-year LC rates of 52.0 and $29.8 \%$, respectively. Hence, in the guidelines of the Chinese Society of Clinical Oncology (CSCO), 50-60 Gy of radiation is the standard dose for dCRT.

As an advantageous radiotherapy technology, SIB-IMRT has been widely used in the treatment of esophageal cancer in recent years. Based on the research results of several phase I/II phase clinical trials [8-11], it is safe and feasible to use SIB-IMRT technology to increase the total dose to a primary tumor to $59.92-70.00 \mathrm{~Gy}$ with a singlefraction dose of 2.14-2.80 Gy. However, whether this technology can provide final local control or a survival benefit to the treatment of esophageal cancer is still questionable due to a lack of sufficient research data [12-14]. Based on the aforementioned research, this study retrospectively analyzed a large number of patients with esophageal cancer treated in a single center of our hospital. For the SIBIMRT group, a lower dose (50.4-60 Gy) was delivered to the PTV P, $_{-}$and the dose delivered to the PTV PT $_{-G}$ was escalated to a higher level (59.92-66.00 Gy). The purpose was to explore whether dose escalation with SIB-IMRT technology might be beneficial in certain esophageal cancer patients. After screening according to the inclusion and exclusion criteria, 375 eligible patients entered the final analysis. It was observed that there were significant differences in the baseline characteristics between the two groups before PSM. The SIB-IMRT group had more women and more cervical and upper thoracic cancers $(P=0.038,<0.001$, respectively). Before case matching, the median PFS of the SD-IMRT and SIB-IMRT groups was 22 and 19 months, respectively, and the median overall survival time was 24 and 22 months, respectively. There was no significant difference in the PFS and OS between the two groups. After case matching, it was found that both in terms of PFS and OS, SIB-IMRT had significant advantages. Compared with SDIMRT, the benefit time of PFS was extended by 5 months, and the benefit time of OS was extended by 6 months. This suggested that SIB-IMRT provided survival benefits for locally advanced esophageal cancer. Further observation of the treatment failure mode of the two groups showed that 
fewer patients in the SIB group experienced locoregional recurrence and distant metastasis: the SIB-IMRT technique tended to show a benefit. It was speculated that the local dose escalation advantage of SIB-IMRT improved the locoregional control of patients, and this then transformed into a long-term survival benefit. The results based on the multivariate analysis also showed that compared with SDIMRT, SIB-IMRT was an independent prognostic factor for long-term patient survival and reduced the patient's risk of death by $39.4 \%$. Based on the above analysis, it can be considered that SIB-IMRT has potential locoregional control and survival benefits for ESCC.

In terms of treatment safety and adverse events, there were no grade 4-5 adverse events in the SIB-IMRT group: the total incidence of grade 3 adverse events was $10.1 \%$ (7/69), of which the incidence of grade 3 radiation esophagitis was $1.4 \%(1 / 69)$. The incidence of grade 3 acute radiation pneumonitis was $2.9 \%$ (2/69), which was similar to the clinically reported data and also showed the safety of SIB-IMRT technology. In the control group there were three cases of grade 4-5 toxicity events, and these were considered to be related to the higher radiation dose exposure of $\mathrm{PTV}_{-\mathrm{C}}$ (the prescribed dose of $\mathrm{PTV}_{-\mathrm{C}}$ in the SDIMRT group was $60 \mathrm{~Gy}$, and that in the SIB-IMRT group was $50.4-60.0 \mathrm{~Gy}$ ).

This study has the following limitations: 1) the study enrollment timespan was large (2003-2014), and there might have existed large differences in technical equipment and treatment factors; 2) although the PSM method was used to balance the differences in the baseline characteristics, and there was no significant difference between the two groups, we could observe that several factors numerically favored the SIB-IMRT group after PSM, e.g., more smaller lesions, fewer patients who received RT alone, etc., which might have skewed the final outcome analysis; 3 ) the observation and recording of treatment-related toxicity may not be sufficiently detailed and accurate, and the late toxicity could not be obtained. Therefore, the conclusions of this study still require further confirmation in prospective studies.

Based on the above results, it was considered that SIBIMRT was safe and reliable compared with SD-IMRT. In addition, SIB-IMRT had locoregional control advantages and potential survival benefits.

Supplementary Information The online version of this article (https:// doi.org/10.1007/s00066-021-01894-y) contains supplementary material, which is available to authorized users.

Acknowledgements We thank LetPub (www.letpub.com) for its linguistic assistance during the preparation of this manuscript.

Author Contribution Wang Lan, Liu lihong: formal analysis, data curation, and writing original draft; Chun Han: writing review and editing, supervision; Liu Shutang, Wang Qi, Xu Liang, Li Xiaoning, Liu Likun: providing medical records and original data.
Conflict of interest W. Lan, L. Lihong, H. Chun, L. Shutang, W. Qi, X. Liang, L. Xiaoning, and L. Likun declare that they have no competing interests.

Open Access This article is licensed under a Creative Commons Attribution 4.0 International License, which permits use, sharing, adaptation, distribution and reproduction in any medium or format, as long as you give appropriate credit to the original author(s) and the source, provide a link to the Creative Commons licence, and indicate if changes were made. The images or other third party material in this article are included in the article's Creative Commons licence, unless indicated otherwise in a credit line to the material. If material is not included in the article's Creative Commons licence and your intended use is not permitted by statutory regulation or exceeds the permitted use, you will need to obtain permission directly from the copyright holder. To view a copy of this licence, visit http://creativecommons.org/licenses/by/4. $0 /$.

\section{References}

1. Franceschini D, Paiar F, Meattini I et al (2013) Simultaneous integrated boost-intensity modulated radiotherapy in head and neck cancer. Laryngoscope 123(12):E97-E103

2. Leclerc M, Maingon P, Hamoir M et al (2013) A dose escalation study with intensity modulated radiation therapy (IMRT) in T2N0, T2N1, T3N0 squamous cell carcinomas (SCC) of the oropharynx, larynx and hypopharynx using a simultaneous integrated boost (SIB) approach. Radiother Oncol 106(3):333-340

3. Jin X, Yi J, Zhou Y et al (2014) A new plan quality index for nasopharyngeal cancer SIB IMRT. Phys Med 30(1):122-127

4. Yi J, Huang X, Gao L et al (2014) Intensity-modulated radiotherapy with simultaneous integrated boost for locoregionally advanced nasopharyngeal carcinoma. Radiat Oncol 9:56

5. Hakariya T, Obata S, Igawa T et al (2014) Feasibility of simultaneous integrated boost IMRT (SIB-IMRT) for castrationresistant prostate cancer. Anticancer Res 34(8):4261-4265

6. Franco P, Arcadipane F, Ragona R et al (2016) Locally advanced (T3-T4 or N) anal cancer treated with simultaneous integrated boost radiotherapy and concurrent chemotherapy. Anticancer Res 36(4):2027-2032

7. Tomasoa NB, Meulendijks D, Nijkamp J et al (2016) Clinical outcome in patients treated with simultaneous integrated boost-intensity modulated radiation therapy (SIB-IMRT) with and without concurrent chemotherapy for squamous cell carcinoma of the anal canal. Acta Oncol 55(6):760-766

8. Li C, Ni W, Wang X et al (2019) A phase I/II radiation dose escalation trial using simultaneous integrated boost technique with elective nodal irradiation and concurrent chemotherapy for unresectable esophageal cancer. Radiat Oncol 14(1):48-56

9. Sakanaka K, Ishida Y, Fujii K et al (2021) Radiation dose-escalated chemoradiotherapy using simultaneous integrated boost intensitymodulated radiotherapy for locally advanced unresectable thoracic esophageal squamous cell carcinoma: a single-institution phase I study. Clin Oncol (R Coll Radiol) 33(3):191-201. https://doi.org/ 10.1016/j.clon.2020.07.012

10. Chen DW, Menon H, Verma V et al (2019) Results of a phase $1 / 2$ trial of chemoradiotherapy with simultaneous integrated boost of radiotherapy dose in unresectable locally advanced esophageal cancer. JAMA Oncol 5(11):1597-1604. https://doi.org/10.1001/ jamaoncol.2019.2809

11. Yu W, Cai YW, Liu Q et al (2015) Safety of dose escalation by simultaneous integrated boosting radiation dose within the primary tumor guided by (18)FDG-PET/CT for esophageal cancer. Radiother Oncol 114(2):195-200 
12. Yu WW, Zhu ZF, Fu XL et al (2014) Simultaneous integrated boost intensity modulated radiotherapy in esophageal carcinoma:early results of a phase II study. Strahlenther Onkol 190(11):979-986

13. Xu YJ, Wang C, Liu G et al (2016) The efficacy and safety of simultaneous integrated boost intensity-modulated radiation therapy for esophageal squamous cell carcinoma in Chinese population: a single institution experience. J Cancer Res Ther 12(1):82-88

14. Hulshof MCCM, Geijsen ED, Rozema T et al (2021) Randomized study on dose escalation in definitive chemoradiation for patients with locally advanced esophageal cancer (ARTDECO study). J Clin Oncol 39(25):2816-2824

15. Ilson DH, Forastiere A, Arquette M et al (2000) A phase II trial of paclitaxel and cisplatin in patients with advanced carcinoma of the esophagus. Cancer J 6(5):316-323

16. Petrash S, Welt A, Reinacher A et al (1998) Chemotherapy with cisplatin and paclitaxel in patients with locally advanced, recurrent or metastatic oesophageal cancer. Br J Cancer 78(4):511-514

17. Ajani JA, Fodor MB, Tjulandin SA et al (2005) Phase II multi-institutional randomized trial of docetaxel plus cisplatin with or without fluorouracil in patients with untreated, advanced gastric, or gastroesophageal adenocarcinoma. J Clin Oncol 23(24):5660-5667

18. Kim JY, Do YR, Park KU et al (2010) A multi-center phase II study of docetaxel plus cisplatin as first-line therapy in patients with metastatic squamous cell esophageal cancer. Cancer Chemother Pharmacol 66(1):31-36

19. Cooper JS, Guo MD, Herskovic A et al (1999) Chemoradiotherapy of locally advanced esophageal cancer: long-term follow-up of a prospective randomized trial (RTOG 85-01). Radiation therapy oncology group. JAMA 281(17):1623-1627

20. Kumar K, Dimri R, Khurana R et al (2007) A randomized trial of radiotherapy compared with cisplatin chemo-radiotherapy in patients with unresectable squamous cell cancer of the esophagus. Radiother Oncol 83(2):139-147
21. Machtay M, Bae K, Movsas B et al (2012) Higher biologically effective dose of radiotherapy is associated with improved outcomes for locally advanced non-small lung carcinoma treated with chemoradiation: an analysis of the radiation therapy oncology group. Int J Radiat Oncol Biol Phys 82(1):425-434

22. Yamoah K, Showalter TN, Ohri N (2015) Radiation therapy intensification for solid tumors: a systematic review of randomized trials. Int J Radiat Oncol Biol Phys 93(4):737-745

23. Minsky BD, Pajak TF, Ginsberg RJ et al (2002) INT 0123 (radiation therapy oncology group 94-05) phase III trial of combined-modality therapy for esophageal cancer: high-dose versus standard-dose radiation therapy. J Clin Oncol 20(5):1167-1174

24. Versteijne E, van Laarhoven HW, van Hooft JE et al (2015) Definitive chemoradiation for patients with inoperable and/or unresectable esophageal cancer: locoregional recurrence pattern. Dis Esophagus 28(5):453-459

25. Teoh AY, Chiu PW, Yeung WK et al (2013) Long term survival outcome after definitive chemoradiation versus surgery in patients with resectable squamous carcinoma of the esophagus: results from a randomized controlled trial. Ann Oncol 24(1):165-171

26. Welsh J, Settle SH, Amini A et al (2012) Failure patterns in patients with esophageal cancer treated with definitive chemoradiation. Cancer 118(10):2632-2640

27. Chen Y, Zhu HP, Wang T et al (2017) What is the optimal radiation dose for non-operable esophageal cancer? Dissecting the evidence in a meta-analysis. Oncotarget 8(51):89095-89107

28. Ren X, Wang L, Han C et al (2018) Retrospective analysis of safety profile of high-dose concurrent chemoradiotherapy for patients with oesophageal squamous cell carcinoma. Radiother Oncol 129(2):293-299 